This is a self-archived version of an original article. This version may differ from the original in pagination and typographic details.

Author(s): Hakala, Ismo; Luomala, Jari

Title: Peer-to-peer cooperative GNSS-based localization for stationary reference nodes in wireless sensor networks

Year: 2017

Version: Accepted version (Final draft)

Copyright: @ IEEE 2017.

Rights: In Copyright

Rights url: http://rightsstatements.org/page//nC/1.0/?language=en

Please cite the original version:

Hakala, I., \& Luomala, J. (2017). Peer-to-peer cooperative GNSS-based localization for stationary reference nodes in wireless sensor networks. In Proceedings of 2017 International Conference on Localization and GNSS (ICL-GNSS) (pp. 1-6). IEEE. International Conference on Localization and GNSS. https://doi.org/10.1109/ICL-GNSS.2017.8376241 


\title{
Peer-to-Peer Cooperative GNSS-based Localization for Stationary Reference Nodes in Wireless Sensor Networks
}

\author{
Ismo Hakala and Jari Luomala \\ University of Jyvaskyla, Kokkola University Consortium Chydenius \\ P.O. Box 567, FI-67701 Kokkola, Finland \\ Email: $\{$ ismo.hakala, jari.luomala $\} @$ chydenius.fi
}

\begin{abstract}
Most localization algorithms in wireless sensor networks rely on a few reference nodes with known locations to estimate the locations of unknown nodes. The locations of reference nodes can be either manually configured or, more practically, obtained by means of some satellite-based positioning $\operatorname{system}(\mathbf{s})$. However, satellite-based locations may be inaccurate and imprecise, which results in reduced location accuracy of localization algorithms. This paper proposes a peer-to-peer cooperative GNSS-based localization algorithm for stationary reference nodes to improve their relative location accuracy and precision. The algorithm applies simple statistical methods and GNSS-based information from multiple reference nodes within a WSN in a peer-to-peer fashion to achieve the improvement. The results of the experiments indicate that both location precision and relative location accuracy are clearly increased due to the algorithm.
\end{abstract}

\section{INTRODUCTION}

The location of sensor nodes is an inherent and valuable information for wireless sensor networks (WSNs). Physical locations can be used to label the sensor data, and are required by many services and applications (e.g., object tracking, geographic routing, location-aware services and applications, etc.) [1], [2]. A simple solution would be to integrate a GNSS receiver with every sensor node to achieve global positions. However, in most cases it would be too expensive in terms of money and power consumption, thus making it impractical. GNSS also has limited availability in some environments, which excludes it from being the only alternative: it does not work indoors, underground, or under dense canopy/foliage, for example. Typically, there are a small number of stationary reference nodes whose locations are known a priori in a WSN, being either manually configured or through some satellitebased positioning system (e.g., GPS). The unknown nodes, i.e., the nodes with unknown locations, deduce their own locations by way of some localization algorithm relying on the reference nodes' locations. However, the known locations obtained through GNSS may be inaccurate and imprecise. The individual location readings of stationary nodes may vary significantly in time (in the order of tens of meters) and space, and may be far from the ground truth location [3]-[5]. The use of these incorrect reference node locations within localization algorithms poses a significant source of error for the location estimates of unknown nodes.
The aforementioned has motivated previous research to find low-cost techniques for mitigating errors and improving the accuracy of stationary GNSS receivers. Some studies focus on improving the accuracy of GNSS nodes directly by using techniques such as wavelet filtering, temporal windowing and spatial clustering [3], while some exploit the variance of GNSS-equipped anchor nodes when locating non-GNSS nodes [5]. Recently, there has been increased interest in cooperative positioning (CP) [6]-[9], where GNSS receivers exchange information with their neighbors to improve performance. Particularly unstructured peer-to-peer (P2P) networks, where data exchange can be done without central control, have many advantages [6].

Wireless sensor networks enable inter-node communication, thus naturally providing a cooperative approach to stationary GNSS error mitigation. By cooperatively exchanging and utilizing GNSS-related information from peer nodes within a local area, together with simple statistics it might be possible to achieve significant improvements without external reference stations or accessing raw satellite data, as high precision GPS systems do (e.g., DGPS, RTK GPS). This would be a costeffective and feasible solution for resource-constrained sensor nodes.

The aim of this paper is to improve both the location precision and relative location accuracy of stationary, low-cost GNSS receivers to be used as references for node localization in outdoor wireless sensor networks. As a solution, we propose a peer-to-peer cooperative GNSS-based localization algorithm for stationary reference nodes in WSNs. It aims to improve the relative location accuracy and precision of reference nodes by applying simple statistical methods and GNSS-based information from various reference nodes within a WSN in a peer-to-peer fashion. It is simple and low-cost in terms of computation, communication, memory and storage allocation, and power consumption. The required $\mathrm{HW}$ can be assembled by using generally available commercial off-the-shelf (COTS) products.

The evaluation results based on the experimental data indicate that the use of the proposed algorithm improves location precision and relative location accuracy. The algorithm is useful for both range-based and range-free node localization if GNSS-based anchor nodes are employed in estimating lo- 
cations. Furthermore, the increased accuracy of the stationary reference nodes can be utilized by a GNSS rover node to fix its location estimates.

To summarize, the main contributions of our study are the following:

- We show the imprecision (variability) and relative inaccuracy of GNSS raw location estimates without any algorithm in use.

- We propose a simple and low-cost P2P cooperative GNSS-based localization algorithm for stationary WSN nodes with GNSS receivers.

- We show through real measurements that the proposed algorithm improves both location precision and relative location accuracy.

The paper has been organized in the following way. Section II provides a brief overview of the main sources of error in satellite-based positioning, and discusses the main quality measures for localization. The experimental measurement and the findings of GNSS raw data are presented in Sections III and IV, respectively. Section V focuses on presenting the proposed GNSS-based localization algorithm. Next, the algorithm is evaluated and analyzed in Section VI. Finally, Section VII concludes the paper.

\section{BACKGROUND}

Global Navigation Satellite Systems (GNSS), such as the U.S. GPS (Global Positioning System) and the Russian GLONASS (GLObalnaya NAvigatsionnaya Sputnikovaya Sistema), enable a receiver to compute its position worldwide within an Earth-fixed reference coordinate frame if it can receive radio signals from at least four navigation satellites [10]. In WSNs, it is often sufficient to know the relative locations of nodes in a particular local coordinate frame instead of global ones. While GNSS is basically intended for producing global coordinates, it can be used to determine local, relative coordinates as well.

The main sources of error in GNSS are satellite clock and ephemeris errors, ionospheric and tropospheric delays, multipath, receiver noise, and errors due to bad satellite constellations (Dilution of Precision, DOP) [10]. Both satellitebased and atmospheric-based errors can be mitigated e.g., by using differential GNSS (DGNSS) because the errors should be equal for all the receivers locally, provided that they use the same satellites in location computation. In contrast, multipath and receiver noise are node-specific and much harder to tackle. Multipath in particular is a bigger problem for stationary nodes than for mobile nodes. The proposed algorithm mainly tries to mitigate the errors caused by multipath and receiver noise which can vary even on a small geographical area.

Location accuracy and precision are the two most important quality measures for evaluating the performance of a localization system [2], [10]. Accuracy is a statistical measure of the distance between the location estimate and the real location, i.e., the ground truth in a particular coordinate system. It is usually expressed as a mean location error. Precision (repeatability) measures the distribution of the location estimates without a relation to the ground truth, i.e., how they are scattered around the mean value.

Different measures can be used to estimate accuracy and precision, such as average location error, distance RMS (DRMS), Circular Error Probability (CEP), and error ellipses [10]. The average location error can be defined as:

$$
L E=\frac{1}{n} \sum_{i=1}^{n} \sqrt{\left(\hat{x}_{i}-x\right)^{2}+\left(\hat{y}_{i}-y\right)^{2}},
$$

where $x$ and $y$ are the ground truth coordinates, $\hat{x}$ and $\hat{y}$ the estimated coordinates, and $n$ the number of location samples. DRMS is defined as [10]:

$$
D R M S=\sqrt{\sigma_{x}^{2}+\sigma_{y}^{2}}
$$

where $\sigma_{x}$ and $\sigma_{y}$ are the root mean square (RMS) values of the errors of the individual axes $x$ and $y$. CEP defines the radius of a circle, centered at the mean value, which includes the location estimates with a probability of $50 \%$. Different confidence levels are also sometimes used. DRMS and CEP are practical methods for expressing precision using a single measure. However, the location error distribution is normally neither circular nor Gaussian, and can be better expressed using error ellipses [10]. The major and minor axes and the rotation angle of the error ellipse can be computed from the covariance matrix of location samples.

\section{EXPERIMENTAL MEASUREMENT}

Our measurement configuration consisted of four Atmel ZigBit $868 \mathrm{MHz}$ sensor nodes (ATZB-900-B0) [11], [12] integrated with a GlobalTop Gms-g6 GNSS module [13], operating as stationary reference nodes. Gms-g6 supports the simultaneous use of both GPS (L1@ @ $1575.42 \mathrm{MHz}$ ) and GLONASS (L1 @ 1598.0625 1605.375 MHz), utilizing the MT3333 GNSS Chipset from MediaTek. Furthermore, our setup comprised of a gateway (Atmel ZigBit $868 \mathrm{MHz}$ and Raspberry Pi 3) and a database server (MongoDB).

The four reference nodes were attached to lamp posts around the university campus parking area at a height of approximately $3 \mathrm{~m}$ by using mounting racks. The distances between the reference nodes were measured using a laser distance meter (Leica DISTO ${ }^{\mathrm{TM}} \mathrm{D} 8$ ). The nodes were batterypowered, featuring an external battery box including two Csize $3.6 \mathrm{~V}$ primary lithium batteries with a nominal capacity of $8.5 \mathrm{Ah}$ (Eve ER26500). The gateway was on the terrace of the campus, and it was mains-powered. The GNSS-WSN setup and node are illustrated in Fig. 1 and 2.

The update rate of the GNSS data used in our experiment was $1 \mathrm{~Hz}$. Once the data was updated, the reference nodes sent the raw data (see TABLE I) to the gateway (see Fig. 1). From the gateway, the raw data was sent to the MongoDB database server to be stored and processed further. MATLAB was used for data processing and analysis.

We performed coordinate transformation from a global geographic coordinate system (lat, lon) to local Cartesian coordinates $(x, y)$ because we were only interested in relative 


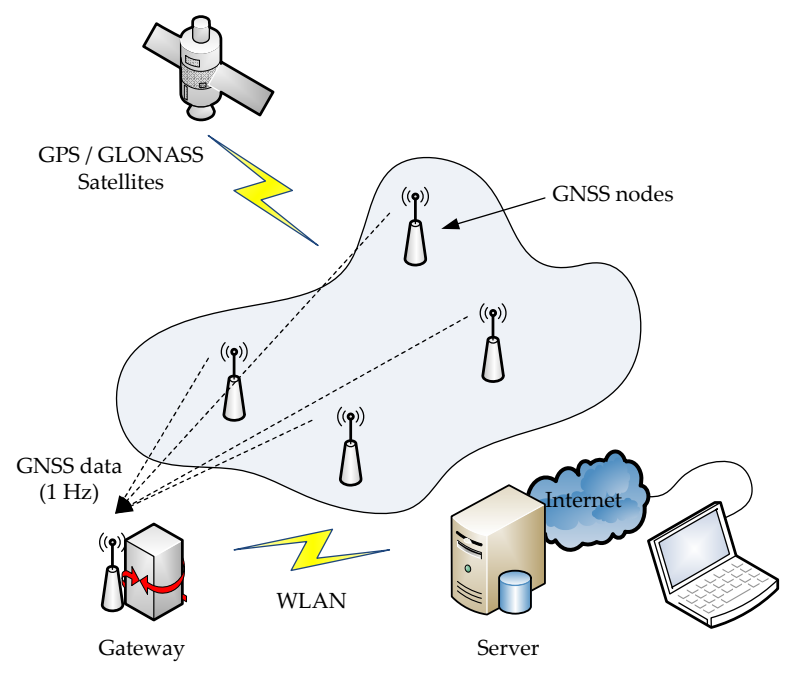

Fig. 1. GNSS-WSN setup for collecting GNSS data.
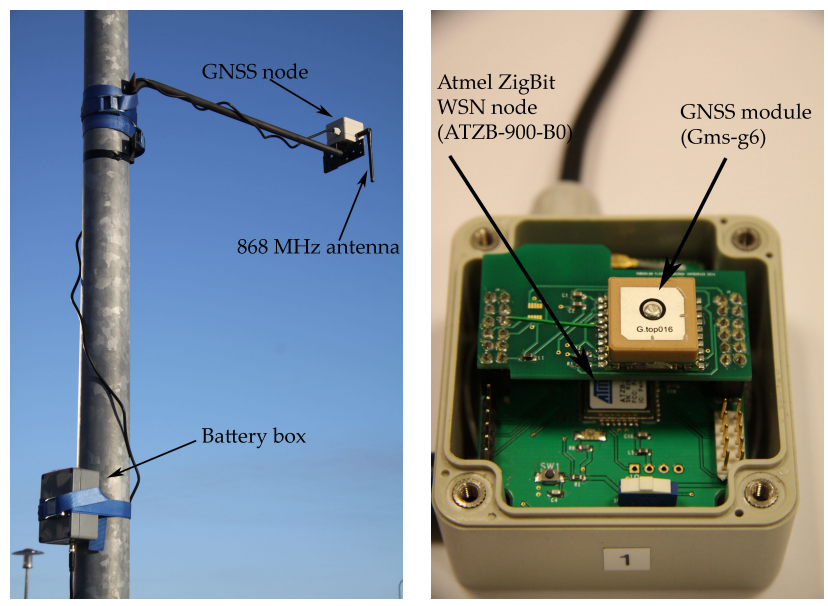

(a)

Fig. 2. (a) GNSS node in the experimental environment, (b) GNSS node's assembly.

TABLE I

COLlected GNSS DATA ( $1 \mathrm{~s}$ SAMPles).

\begin{tabular}{ll}
\hline Data & Description \\
\hline \hline UTC Time & hhmmss \\
Latitude & ddmm.mmmm \\
Longitude & (d)ddmm.mmmm \\
PDOP & Position Dilution of Precision \\
HDOP & Horizontal Dilution of Precision \\
VDOP & Vertical Dilution of Precision \\
Satellites Used & $0-24$ \\
Satellite Used & List of active satellites (IDs) used in localization \\
Position Fix Indicator & $0-2(0=$ Fix NA, $1=$ GPS fix, $2=$ DGPS fix $)$ \\
Operating mode 1 & M/A $(\mathrm{M}=$ Manual, A = Automatic) \\
Operating mode 2 & $1-3(1=$ Fix NA, $2=2 \mathrm{D}, 3=3 \mathrm{D})$ \\
\hline
\end{tabular}

locations. First, the latitude/longitude samples were converted from degrees and minutes to decimal degrees (DD). Next, the $l_{a} t_{D D}$ and $\operatorname{lon}_{D D}$ were converted to local Cartesian
TABLE II

Average/Std of DOPS AND Number of SATElLites (1 s DATA)

\begin{tabular}{l|c|c|c|c}
\hline Node & PDOP & HDOP & VDOP & \#Sat. \\
\hline \hline 1 & $1.14 \pm 0.12$ & $0.59 \pm 0.05$ & $0.97 \pm 0.12$ & $19.12 \pm 1.57$ \\
2 & $1.12 \pm 0.12$ & $0.59 \pm 0.05$ & $0.95 \pm 0.12$ & $19.47 \pm 1.46$ \\
3 & $1.13 \pm 0.12$ & $0.59 \pm 0.05$ & $0.97 \pm 0.12$ & $19.15 \pm 1.59$ \\
4 & $1.14 \pm 0.12$ & $0.59 \pm 0.05$ & $0.97 \pm 0.11$ & $19.02 \pm 1.54$ \\
\hline
\end{tabular}

coordinate system as follows:

$$
\begin{aligned}
& x=\left(\operatorname{lon}_{D D}-X_{0}\right) \cdot\|D D\| \cdot \cos \left(\operatorname{lat}_{D D} \cdot \frac{\pi}{180}\right) \\
& y=\left(\operatorname{lat}_{D D}-Y_{0}\right) \cdot\|D D\|
\end{aligned}
$$

where $\left(X_{0}, Y_{0}\right)$ is the location of the defined origin (in DD) in the local coordinate system, and $\|D D\|=111320$ is the length of a decimal degree $[\mathrm{m}]$ at the equator. We set the origin to the mean location of node 3 .

\section{VARIATION OF EXPERIMENTAL GNSS RAW DATA}

The experimental GNSS raw data analyzed here was collected by WSN nodes during nine days between February 4th and 12th, 2017. The amount of collected data samples per node was around 770000 on average, denoting roughly a 97\% Packet Reception Ratio (PRR) in WSN. We examine the achieved location accuracy and precision of the GNSS nodes, as well as the satellite geometry at the particular time.

Dilution of Precision (DOP) is a measure of geometry of the solution derived from the relative locations of the receiver and the satellites which were used to compute its location [10]. DOP is a unitless number, and can be seen as a multiplier of the predicted error. Thus, a smaller DOP value means better geometry for location estimation. The different measures of DOP and the number of satellites used for location computation in our experiment are shown in Table II. As can be seen, both the DOP values and the number of satellites are relatively good. They are both also of the same size for all the nodes. This indicates that the satellite constellation does not cause a significant error in the location estimates, and this error is approximately of the same size for all nodes.

Our experiments confirmed that the individual location readings of GNSS receivers vary quite heavily, both temporally and spatially. They drift both in the short-term (e.g., within a few hours) and long-term (e.g., within a few days) measurements. The fluctuation between the nodes can also differ in size and direction. This is illustrated in Fig. 3. In Fig. 3(a), the $1 \mathrm{~s}$ location estimates $(x, y)$ of each GNSS node are plotted over the measurement period (nine days). The computed CEP $(50 \%)$ values for the nodes are between $2.08 \mathrm{~m}$ and $2.83 \mathrm{~m}$. The 'accuracy' ( $50 \%$ CEP) reported by the manufacturer is $3.0 \mathrm{~m}$ for GPS and $2.5 \mathrm{~m}$ for DGPS [13].

Fig. 3(b) shows the deviation of each $1 \mathrm{~s}$ GNSS reading from the mean during $3 \mathrm{~h}$, according to (1), averaged over the nodes. This indicates that the individual GNSS readings are imprecise, i.e., they fluctuate or drift around the mean value. As shown in Fig. 3(c), the inter-node distance errors 


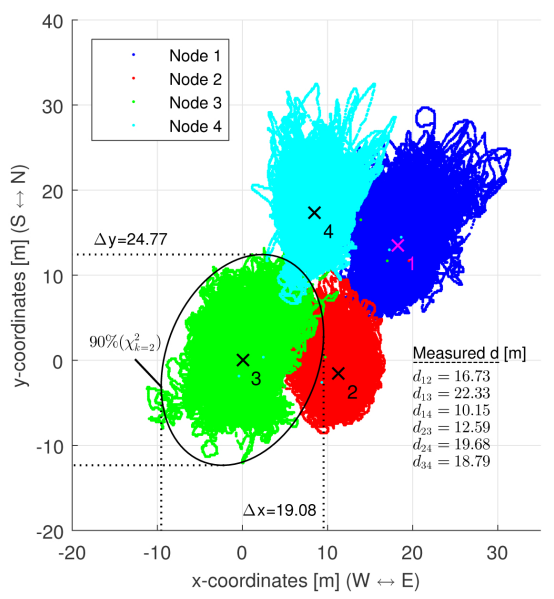

(a)

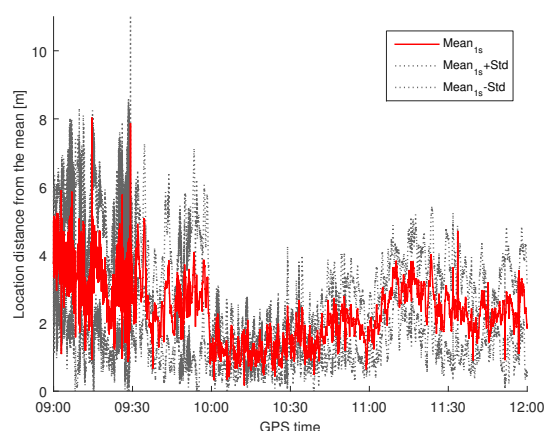

(b)

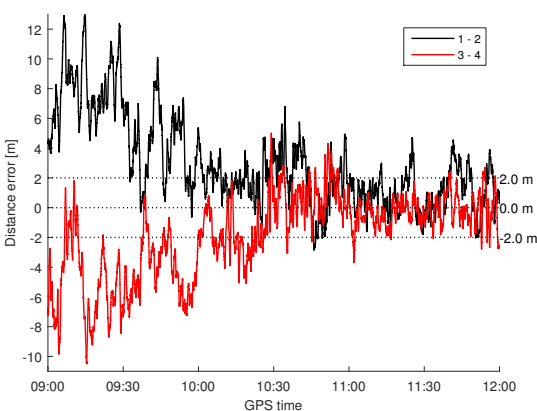

(c)

Fig. 3. (a) GNSS location estimates (1 s samples and mean) during a measurement period of 9 days $(\bar{N}=769858)$, (b) location precision, i.e. the distance of nodes' $1 \mathrm{~s}$ samples $(x, y)$ from their $3 \mathrm{~h}$ mean (Mean and standard deviation of nodes), (c) an example of inter-node distance errors from two distances based on $1 \mathrm{~s}$ samples. Measurements (b, c) taken from a period of $3 \mathrm{~h}$ on February 5th, 2017.

computed based on nodes' coordinates also fluctuate over time. Therefore, the GNSS readings are inaccurate, too.

\section{GNSS-BASED LOCALIZATION ALGORITHM FOR STATIONARY REFERENCE NODES}

Based on the experimental data, we propose the following algorithm as our first draft to improve the location accuracy and precision of stationary GNSS-equipped reference nodes in WSNs. As an input for the algorithm, we use $1 \mathrm{~min}$ average locations $(x, y)$. The algorithm itself comprises of three separate phases, described in the following subsections. The flow chart of the algorithm is depicted in Fig. 4.

1) Smoothing Location Estimates: The detection and appropriate processing of potential outliers in the raw location data is a pre-processing phase that should be done prior to any other processing. For detecting outliers, nodes compute DRMS values for the location data cumulatively from the beginning. If the location error of the $1 \mathrm{~min}$ sample $i$ is greater than the 2DRMS value, then the sample $i$ shall be replaced with the cumulative mean of the location data. After outlier mitigation, to further smooth out the location estimates, nodes compute 10 min simple moving averages (SMA) based on the $1 \mathrm{~min}$ average values.

2) Adjusting Location Estimates: To utilize location-related information from the entire WSN area, we employ all the reference nodes to compute a network-specific weighted differential correction to be used as an adjustment for individual location estimates. The weighted differential correction (WDC) for both $x$ and $y$ coordinates is computed as follows:

$$
W D C=\frac{\sum_{j=1}^{n} w_{j} e_{j}}{\sum_{j=1}^{n} w_{j}},
$$

where the weight $w_{j}=1 / \sigma_{j}, \sigma_{j}$ is the cumulative standard deviation of the reference node $j$ location samples $\left(x_{1 \min }, y_{1 \min }\right)$ at the given time, $e_{j}$ the corresponding distance error between the sample location (after smoothing) and the

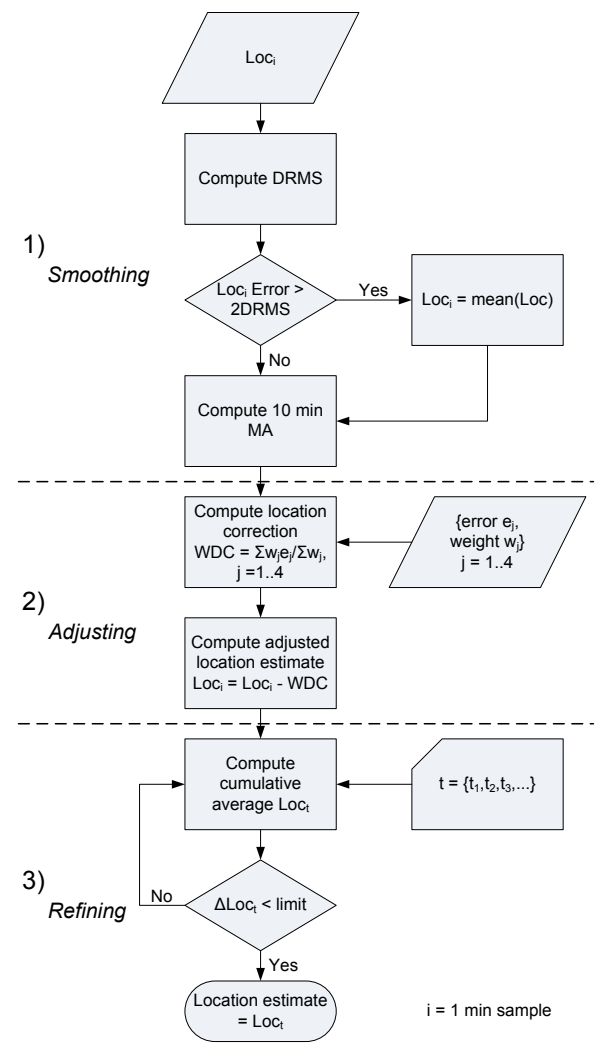

Fig. 4. GNSS-based localization algorithm for stationary reference nodes.

cumulative mean location (of adjusted data), and $n$ the number of GNSS reference nodes ( 4 in our case). In order to compute the adjustment, each reference node broadcasts its weight $w_{j}$ and distance error $e_{j}$ once per minute to the other reference nodes. By applying the adjustment, the new location estimate $L o c_{i}$ for the sample $i$ is computed as follows:

$$
L o c_{i}=L o c_{i}-W D C .
$$


The aim of this procedure is to try and minimize the effect of individual nodes' GNSS errors, due to e.g. multipath and receiver noise, by exploiting multiple nodes cooperatively and emphasizing the nodes with smaller deviations. If only one reference node was used in the correction (like in DGNSS), its placement should be carefully planned and its receiver noise level should be low; conditions which cannot always be fulfilled.

3) Refining Location Estimates: Despite the smoothing and data adjustment, location estimates (1 $\mathrm{min})$ still vary quite a lot, although the deviation is more than halved from the 1 min raw data. This is probably a result of the GNSS error causes discussed earlier. To overcome this issue we use statistical methods and compute the cumulative moving average (CMA) of location estimates. At the predefined time periods $t=\left\{t_{1}, t_{2}, t_{3}, \ldots\right\}$, nodes could check if the difference between the last two CMA values is smaller than a predefined limit. If it is, the last CMA value is set as the final location estimate; otherwise the process continues until the condition is fulfilled. Alternatively, the process could be continued to the predefined time $t$ without comparisons to any limit. The values of the time periods $t_{i}$ and the refinement limit are implementation-specific and can be defined as needed.

\section{Performance EVAluation AND Discussion}

We evaluated the performance of the proposed algorithm with MATLAB by using the same measurement data as above in the computations. We analyze the location estimates achieved using the algorithm and compare them with the 1 min average GNSS data regarding location precision and relative location accuracy.

\section{A. Evaluation of Location Precision}

The algorithm clearly improves location precision, as can be seen in Fig. 5(a), where the GNSS location estimates after the algorithm phases $1 \& 2$ are plotted over the measurement period (Raw data on the background in grey). The CEP (50\%) of the GNSS raw data $(1 \mathrm{~min})$ for the nodes is between $1.99 \mathrm{~m}$ and $2.75 \mathrm{~m}$, and between $0.78 \mathrm{~m}$ and $1.14 \mathrm{~m}$ after the algorithm phases $1 \& 2$, resulting in an improvement of about $60 \%$.

To further evaluate the precision of location estimates, we computed DRMS for different averaging times. The results for the measurement period are summarized in Fig. 5(b). As can be seen, location precision is improved (the DRMS decreases) while the averaging time increases. After 1 hour, DRMS, averaged over the nodes, has fallen below 1 meter and keeps improving. It should be noted that the number of samples decreases while the averaging time increases. Compared to the 1 min raw data, the improvement achieved by using the algorithm is quite significant. The improvement is caused by the joint effect of smoothing and adjusting location estimates (the algorithm phases $1 \& 2$ ), as can be seen in Fig. 5(b). The DRMS values of the $1 \mathrm{~min}$ data for the individual nodes are presented in Table III.
TABLE III

DRMS OF NODES [M]

\begin{tabular}{l|c|c|c}
\hline Node & Raw $(1 \mathrm{~min})$ & Alg. $(1 \mathrm{~min})$ & $\mathrm{n}$ \\
\hline \hline 1 & 3.22 & 1.27 & 12739 \\
2 & 2.40 & 0.94 & 12738 \\
3 & 3.30 & 1.36 & 12741 \\
4 & 2.71 & 1.07 & 12740 \\
\hline Mean & 2.91 & 1.16 & 12740 \\
\hline
\end{tabular}

TABLE IV

INTER-NODE DISTANCE ERRORS [M]

\begin{tabular}{l|r|r|r|r|r|r|r|r}
\hline Distance & $1 \mathrm{~h}$ & $3 \mathrm{~h}$ & $6 \mathrm{~h}$ & $12 \mathrm{~h}$ & $1 \mathrm{~d}$ & $2 \mathrm{~d}$ & $4 \mathrm{~d}$ & $8 \mathrm{~d}$ \\
\hline \hline $1-2$ & 0.60 & 0.59 & 0.09 & 0.11 & -0.21 & -0.14 & -0.11 & -0.12 \\
$1-3$ & -0.05 & -0.58 & -0.37 & -0.28 & 0.20 & 0.46 & 0.36 & 0.39 \\
$1-4$ & 0.42 & -0.88 & -0.39 & -0.32 & 0.14 & 0.34 & 0.33 & 0.29 \\
$2-3$ & -2.30 & -2.13 & -1.76 & -1.71 & -1.43 & -1.17 & -1.18 & -1.13 \\
$2-4$ & -0.88 & -0.80 & -0.97 & -0.91 & -0.66 & -0.58 & -0.51 & -0.58 \\
$3-4$ & -0.54 & -0.46 & -0.34 & -0.26 & 0.55 & 0.61 & 0.54 & 0.51 \\
\hline MAE & 0.80 & 0.91 & 0.65 & 0.60 & 0.53 & 0.55 & 0.50 & 0.50 \\
\hline
\end{tabular}

\section{B. Evaluation of Location Accuracy}

The absolute location accuracy could not be evaluated since we did not know the real locations of the reference nodes. However, we did know the real measured inter-node distances, and we could use the mean absolute inter-node distance error as an approximate measure of relative location accuracy/error. To approximate the relative location error on a WSN level, we computed the mean absolute inter-node distance error (MAE) as follows:

$$
M A E=\frac{2}{n(n-1)} \sum_{i=1}^{n-1} \sum_{j=i+1}^{n}\left|\hat{d}_{i, j}-d_{i, j}\right|,
$$

where $\hat{d}_{i, j}$ is the inter-node distance estimate for the nodes $i$ and $j$ computed based on the estimated node locations, $d_{i, j}$ the corresponding measured distance, respectively, and $n$ the number of reference nodes $(n=4)$. It is hypothesized that the smaller the MAE, the smaller the relative location error is on a WSN level.

The results are shown in Fig. 5(c). As can be seen, the MAE decreases while the averaging time increases. Therefore, it can be assumed that the relative location accuracy of the nodes improves on average, yielding sub-meter accuracy. The improvement in relative location accuracy is mainly caused by refining location estimates (the algorithm phase 3). Smoothing/adjusting location estimates seems to have only minor effect on location accuracy. This is reasonable since the correction is of the same size for all the nodes, thus keeping the geometric shape of the network unchanged. Also, the aim of moving average is to smooth out the peak values, which does not affect the mean values much.

The inter-node distance errors of individual distances are presented in Table IV. As can be seen, some distances are very accurate (e.g., $1-2$ ) while some exhibit more error (e.g., $2-3$ ). This might be caused by a bias in some node's or nodes' location due to a number of possible reasons, possibly due to multipath propagation. 


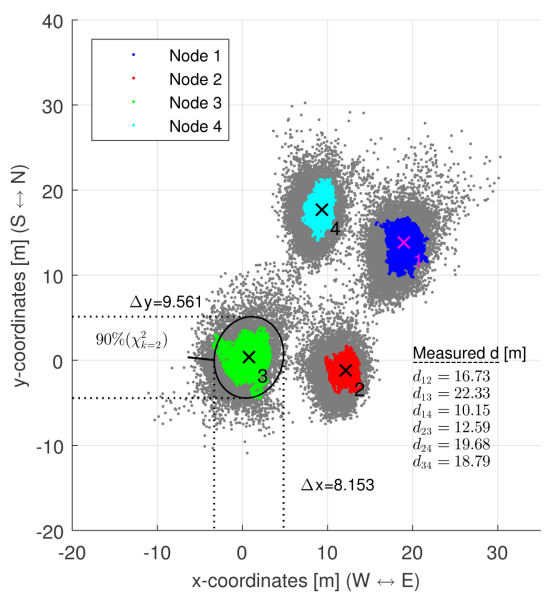

(a)

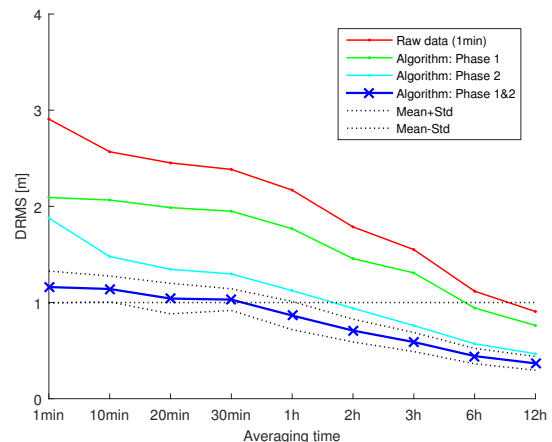

(b)

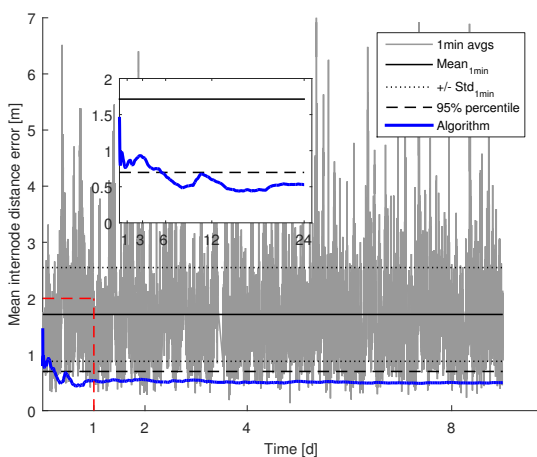

(c)

Fig. 5. (a) GNSS location estimates ( 1 min samples and mean) after the algorithm phases $1 \& 2$ (1 min raw data in grey), (b) the location precision (Distance RMS) of the algorithm for different averaging times (Mean and standard deviation of nodes), (c) the mean of absolute inter-node distance errors. Measurement period of 9 days.

\section{Discussion}

Based on the experimental data, the proposed algorithm clearly improves both the location precision and relative location accuracy of stationary GNSS nodes. This will result in better performance of anchor-based node localization in WSNs. The deviation of location estimates decreases immediately, thus making the results more reliable. In our setup, after $1 \mathrm{~h}$, DRMS/CEP is below $1 \mathrm{~m}$ on average, which is at an adequate level for many requirements. However, it is good to bear in mind the possible sources of error, such as the resolution of the GNSS receiver and the inter-node distance measurements, which introduce uncertainty to the results. In our experimental measurement, the purpose was to collect a large amount of GNSS data continuously during several days. Therefore, the power consumption was relatively high. Reference nodes do not often rely on primary batteries. However, power consumption can be reduced in order to get them to operate as battery-powered. In a real implementation, the update rate could be slower and the measurement period shorter to reduce power consumption, yet resulting in an improvement of about the same size based on our preliminary tests. Lastly, the computation, communication, memory and storage allocation required by the algorithm is reasonable. It can be easily adopted in WSNs and implemented into typical resource-constrained, low-cost WSN nodes without floatingpoint arithmetic needed.

\section{CONCLUSION}

This paper has proposed a peer-to-peer cooperative GNSSbased localization algorithm for stationary reference nodes in wireless sensor networks. The main goal of the algorithm is to improve the location precision and relative location accuracy of low-cost GNSS receivers. The results indicate that the algorithm increases both location precision and relative location accuracy by employing simple, low-cost methods and peer-to-peer communication. Therefore, it can be practi- cally implemented into resource-constrained WSN nodes. The proposed algorithm could be widely utilized in GNSS-based localization, particularly node localization in WSNs.

\section{REFERENCES}

[1] N. Patwari, J. Ash, S. Kyperountas, A. Hero III, R. Moses, and N. Correal, "Locating the Nodes: Cooperative localization in wireless sensor networks," IEEE Signal Process. Mag., pp. 54-69, July 2005.

[2] W. Dargie and C. Poellabauer, Fundamentals of Wireless Sensor Networks: Theory and Practice, ser. Wiley Series on Wireless Communications and Mobile Computing. John Wiley \& Sons Ltd., 2010.

[3] M. Aram, B. Li, S. Krishnan, A. Anpalagan, and B. Grush, "Improving Position Estimates from a Stationary GNSS Receiver using Wavelets and Clustering," in Canadian Conference on Electrical and Computer Engineering (CCECE'06), Ottawa, Canada, May 2006, pp. 758-762.

[4] Y. Zhou, J. Schembri, L. Lamont, and J. Bird, "Analysis of Stand-alone GPS for Relative Location Discovery in Wireless Sensor Networks," in Canadian Conference on Electrical and Computer Engineering (CCECE'09), St. John's, Canada, May 2009, pp. 437-441.

[5] B. Cheng, R. Du, B. Yang, W. Yu, C. Chen, and X. Guan, "An Accurate GPS-based Localization In Wireless Sensor Networks: A GMWLS Method," in 40th International Conference on Parallel Processing Workshops (ICPPW), Taipei City, Taiwan, September 2011, pp. 33-41.

[6] R. Garello, L. Lo Presti, G. Corazza, and J. Samson, "Peer-to-Peer Cooperative Positioning - Part I: GNSS-Aided Acquisition," InsideGNSS, vol. 7, no. 2, pp. 55-63, March/April 2012.

[7] F. Penna, M. Caceres, and H. Wymeersch, "Cramér-Rao Bound for Hybrid GNSS-Terrestrial Cooperative Positioning," IEEE Commun. Lett., vol. 14 , no. 11 , November 2010.

[8] F. Dovis, C.-F. Chiasserini, L. Musumeci, and C. Borgiattino, "Contextaware Peer-to-Peer and Cooperative Positioning," in International Conference on Localization and GNSS (ICL-GNSS 2014), Helsinki, Finland, June 2014.

[9] N. Alam, A. Balaei, and A. Dempster, "Performance Boundaries for Cooperative Positioning in VANETs," in International Global Navigation Satellite Systems Society IGNSS Symposium 2011, Sydney, Australia, November 2011.

[10] D. Bartlett, Essentials of Positioning and Location Technology, ser. The Cambridge Wireless Essentials Series. Cambridge University Press, 2013.

[11] Atmel Corporation, ZigBit ${ }^{T M} 700 / 800 / 900 \mathrm{MHz}$ Wireless Modules ATZB-900-BO Datasheet, 2009.

[12] — Low Power 700/800/900 MHz Transceiver for IEEE 802.15.42006, IEEE 802.15.4c-2009, Zigbee, 6LoWPAN, and ISM Applications - AT86RF212, 2010.

[13] GlobalTop Technology Inc., Gms-g6 GNSS Module Datasheet, Rev. V0C ed., 2013, URL: www.gtop-tech.com. 